\title{
A simple urine-collecting apparatus and method for cows and heifers
}

\author{
Z. J. Cao, ${ }^{*}$ M. Ma, ${ }^{*}$ X. Y. Yan, † S. L. Li, ${ }^{*}$ and X. M. Zhang*1 \\ *State Key Laboratory of Animal Nutrition, College of Animal Science and Technology, China Agricultural University, Beijing, China 100193 \\ †College of Animal Science, South China Agricultural University, Guangzhou, China 510642
}

\begin{abstract}
An apparatus and method that can allow continuous and precise total collection of urine under normal conditions was developed that could satisfy the urinesampling requirement of balance studies, metabolic experiments, and hormone trials of cows and heifers. The apparatus is composed of an anterior urethra, a urine cup, and a collection barrel. Total and precise collection of urine can be achieved without leakage, fecal contamination, or urethra infection. The apparatus has few negative effects on the cows and does not influence the cows' normal physiological activities.
\end{abstract}

Key words: urine collection, apparatus, method

\section{INTRODUCTION}

Urine collection is one of the most basic procedures in animal experiments such as hormone trials and nitrogen balance studies. It is also a crucial factor that can influence the accuracy of energy metabolism experiments. Because of the complicated structure of the urinary anatomy, there has previously been no perfect apparatus and method to collect urine continuously and precisely from cows and heifers without having negative effects on their health and performance. This is also one of the reasons that bulls and steers are used as replacements in many experiments. Scientific results may not be applicable because of the huge differences in the aspects of physiological structure, nutrient requirements, and metabolic characteristics between cows and bulls.

In the past, manual collection of urine has been used, with great labor cost. This has prevented cows from being fed in a normal environment and has probably affected the reference for urine volume. Forbes et al. (1937) described an apparatus in which a rubber tube was strapped over the vulva by using a harness transfer for conducting the urine to a container. Hobbs et al. (1950) improved the above apparatus by using branding cement to attach the straps and tube to the cows. The

Received January 15, 2009.

Accepted May 13, 2009

${ }^{1}$ Corresponding author: xiaomingzh@cau.edu.cn
2 similar apparatuses could collect urine effectively, but removal of the devices was rather difficult and caused raw spots devoid of hair where the straps were fastened with branding cement. Furthermore, during the standing up and lying down activities of cows, there was a potential risk of leaking urine because the apparatus did not have a totally closed urine collection tube. Balch et al. (1951) modified the apparatus by using a harness with a feces-collection bag and a rubber tube to conduct urine into a container placed on the floor. Gorski et al. (1957) reported an apparatus that collected urine and feces concurrently, which provided mobility by using a portable urine bag and a smaller, lighter weight feces bag based on the model by Balch et al. (1951). A urine cup for long-term collection of urine from cows, using glue to place straps in position, was described by Fellner et al. (1988). However, the apparatus required frequent supervision and rearrangement because the device and feces weighed too much. The method gave good results only with some cows. Additionally, it was easier to collect feces than urine. Although the different methods described above had many advantages, there were also some major disadvantages, such as the complexity of the apparatus, the difficulty of handling procedures, and the negative effects on cows. The objective of this paper is to describe an easy-to-handle method and an inexpensive, portable, and reusable apparatus to collect urine continuously and precisely, with few negative effects on the cows.

\section{MATERIALS AND METHODS}

Animal care and use were approved and conducted under established standards of the College of Animal Science and Technology, China Agricultural University, Beijing, China.

\section{Anterior Urethra}

The anterior urethra was constructed from a rubber extension glove with a $0.1-\mathrm{cm}$ thickness. The fingers were first removed by cutting and trimming the glove to the required dimensions of the anterior urethra (Figure 1). From the side view, the anterior urethra was cone-shaped, with a large end and a small end. 


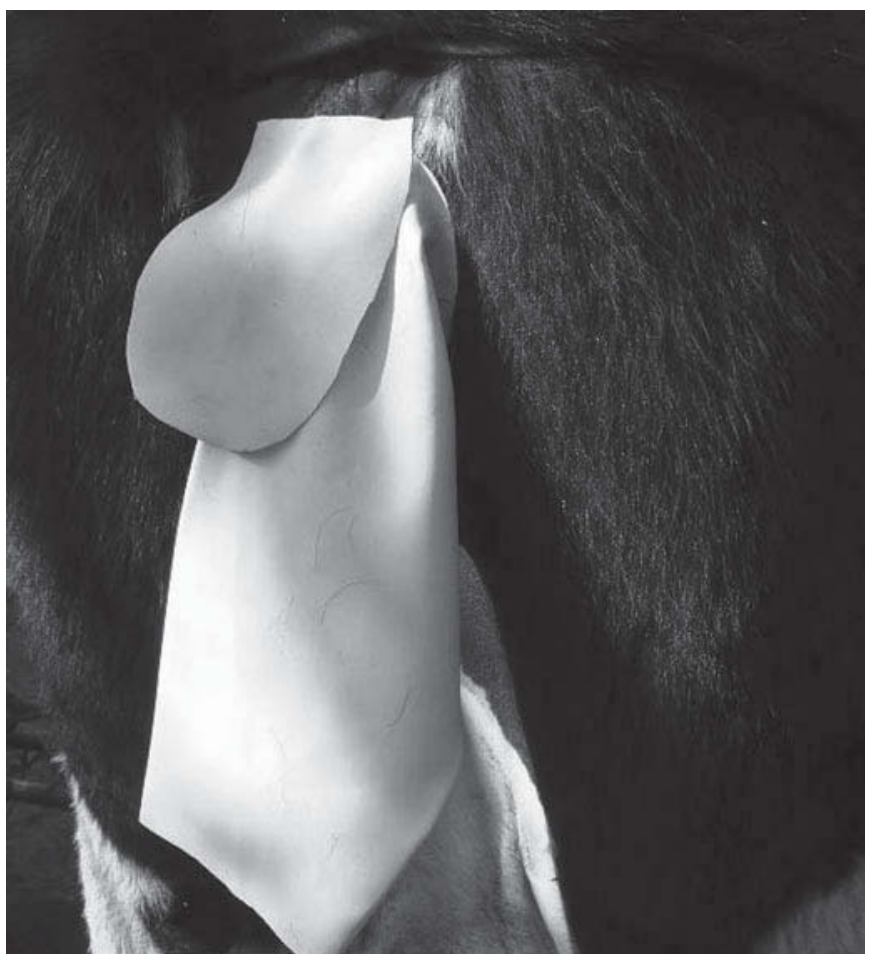

Figure 1. Appearance of the anterior urethra.

The large upper diameter of the anterior urethra was approximately $15 \mathrm{~cm}$; this should be a suitable size to cover the edge of the external genitalia area entirely. The small lower diameter of the anterior urethra was about $8 \mathrm{~cm}$, and this must be in close contact with the urine cup. The length of the anterior urethra was 20 $\mathrm{cm}$. The total anterior urethra would be covered by the urine cup

\section{Urine Cup}

The urine cup was composed of an infundibular rubber cup, canvas, drainage hose, and straps (Figure 2). Bicycle or car tire inner tubing of 0.2-cm thickness, attached to the distal end with rubber cement, could be used as the primary material for the infundibular rubber cup. According to the shape of the infundibular rubber cup, canvas, with a thickness of $0.2-\mathrm{cm}$, was cut to dimensions large enough to cover the vulva areas of the cows and was sewn onto the infundibular rubber cup with plastic thread. At the same time, 8 circular holes were made in the canvas to fix the straps onto the cows. The straps were made of flexible rubber hose with a 1.0-cm inside diameter and a $0.2-\mathrm{cm}$ thickness, which could ensure that the pressure was suitably distributed and that there were no injuries to the body, especially to the mammary vein. The large upper diameter of the urine cup was approximately $40 \mathrm{~cm}$, which should be

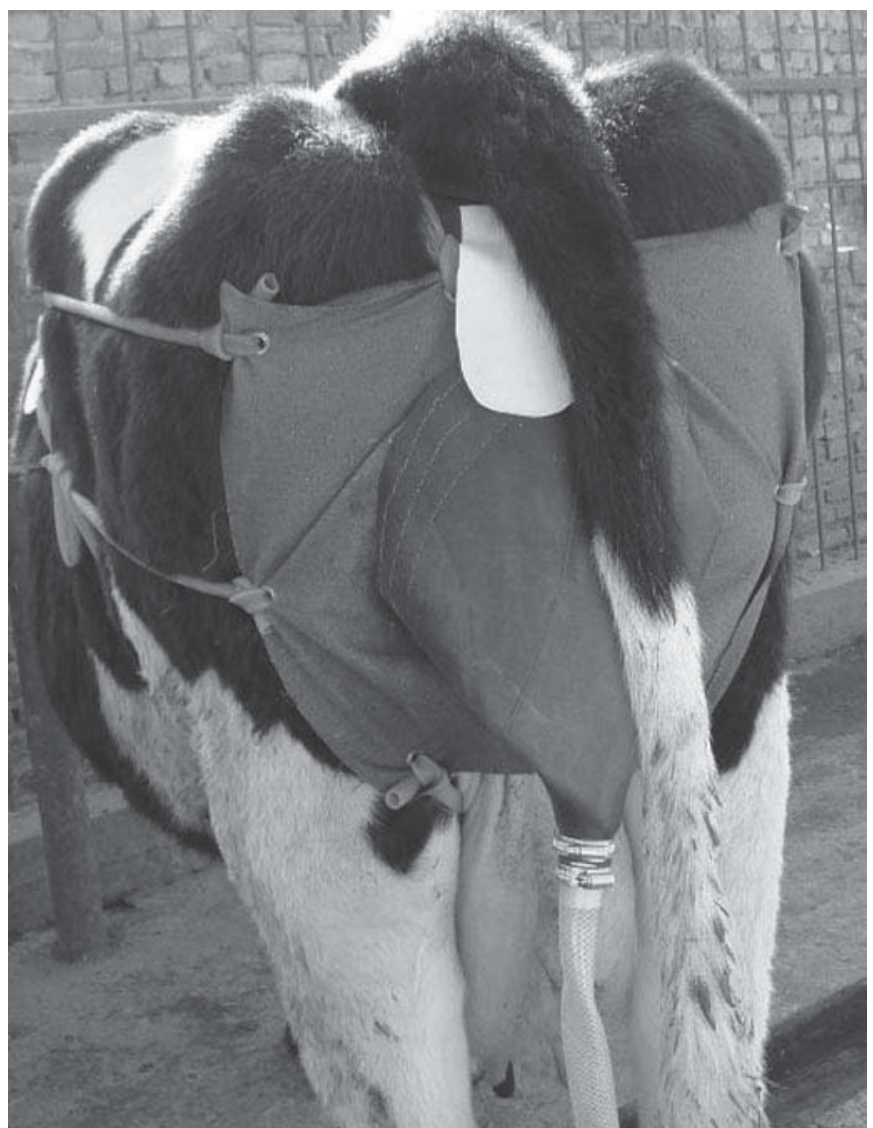

Figure 2. Appearance of the urine cup.

a suitable size to cover the edge of the buttocks area entirely. The small lower diameter of the urine cup was about $3.0 \mathrm{~cm}$, and this must be in close contact with the drainage hose. A 3.0-cm diameter drainage hose made of transparent polyvinyl chloride flexible pipe was then fixed onto the small lower end of the urine cup by means of steel wires, to conduct the urine from the urine cup to the collection barrel. Suitable cleanness with detergent and rinsing in hot water after each collection period would ensure that the urine cup was reusable many times.

\section{Collection Barrel}

A barrel made of transparent hard plastic would be a good choice as a collection barrel. The diameter of the collection barrel opening should be approximately $3.5 \mathrm{~cm}$ to ensure that the drainage hose can be inserted into the barrel. The urine was removed and collected from the barrel twice each day in most of the experiments; therefore, the volume of the collection barrel should be about $20 \mathrm{~L}$ because cows can produce 9.1 to $35.7 \mathrm{~L} / \mathrm{d}$ of urine. 


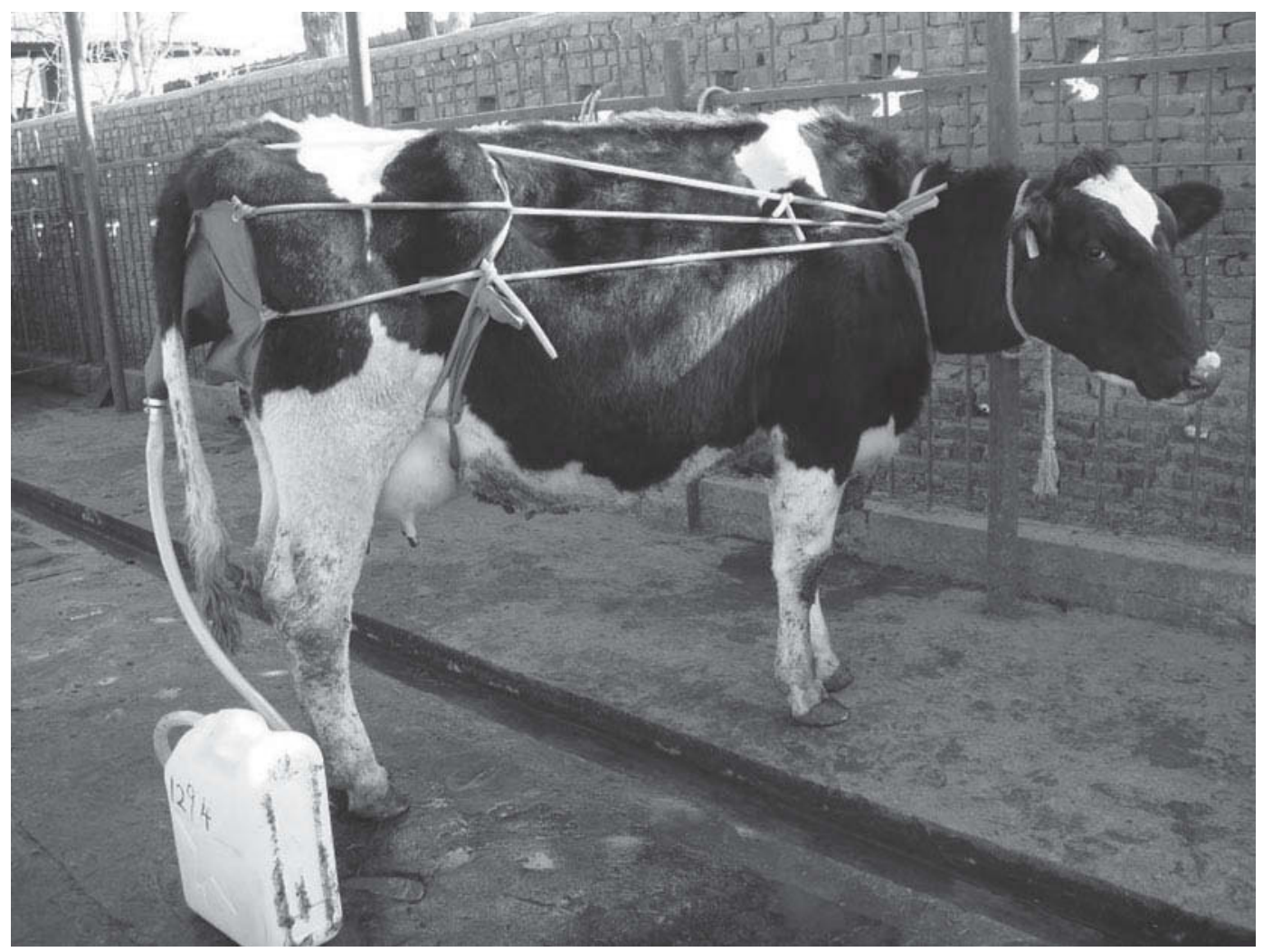

Figure 3. Appearance of the apparatus.

\section{Installation of Urine Apparatus}

The external genitalia area was cleaned with soap, rinsed, and dried. The anterior urethra was cut to suitable dimensions according to the size of the urogenital tract plicae. The anterior urethra was spread with glue (No. 502, 502 Glue Factory, Beijing, China) and adhered to the urogenital tract plicae with appropriate pressure for $5 \mathrm{~s}$. The anterior urethra was then successfully fixed onto the cow. A septum cut from the glove was attached with adhesive to the upper external genitalia to avoid fecal contamination of the anterior urethra.

The anterior urethra can remain in place for at least $5 \mathrm{~d}$ and will fall off the cows automatically with the loss of adhesion of the glue. There is no need to remove it manually, which minimizes the damage to the urogenital tract plicae. The apparatus should be checked periodically during the experimental period so that any maladjustment can be corrected before urine contamination takes place.

Two canvas straps were yoked to the neck and chest of the cows before the urine cup was harnessed in position to cover the anterior urethra (Figure 3). Eight flexible rubber hose straps were then pulled into position across the cows' back, between the backbone and hipbone, un- der the belly, and between the funnel and the feet. The locations for rear patches were first traced, followed by tracing the rump and thigh patches, respectively. Rump straps were placed alongside the tail at a position that seemed to cause the least friction and irritation of the tail or pin bones. Thigh straps were placed symmetrically on either side. The additional cords going between the hind legs of the cows from the lower rings of the urinal to the straps under the belly were tied.

The 10-cm distal end of the drainage hose was inserted into the opening of a $20-\mathrm{L}$ urine collection barrel and fastened with a steel wire. A 500-mL quantity of $10 \% \mathrm{H}_{2} \mathrm{SO}_{4}$ was added to the container to avoid nitrogen loss during the studies. The collection barrel was placed behind the cow on the floor. Under conditions of extreme cold, the drainage hose could be wrapped with a thick pad to avoid the urine freezing.

\section{RESULTS AND DISCUSSION}

The method creates a fully enclosed "tube" from the meatus urinarius to the collection barrel. Urine can be totally collected without leakage and fecal contamination, ensuring accurate results when the apparatus and method are used correctly. 
The anterior urethra may be kept in place for at least $5 \mathrm{~d}$. This period is sufficient to collect urine for most experiments. The viscosity of different glues is the main influential factor in the length of time the anterior urethra stays in place, whereas the durability of adhesion seems dependent on various factors, including skin moisture, skin oils, cleanliness, exposure to water, cow movement, and, most important, correct placement of the anterior urethra (Fellner et al., 1988).

The anterior urethra is made of soft materials and has a large contact area that does not cause injury to the body. In addition, the apparatus does not affect the cows' normal physiological activities, such as eating, ruminating, being milked, urinating, standing up, and lying down. To date, approximately 200 apparatuses have been constructed using the above-mentioned materials, and this method has been used for urine collection in balance studies, metabolic experiments, and hormone trials at 6 universities. In our studies, Holstein cows using this apparatus averaged $15.7 \mathrm{~L} / \mathrm{d}$ of urine $(\mathrm{SD}=4.9)$. Urine volumes ranged from 9.1 to 35.7 $\mathrm{L} / \mathrm{d}$ for cows producing from 11.2 to $40.5 \mathrm{~kg} / \mathrm{d}$ of milk during the experimental period, respectively. The total amount of urine produced by any one cow varied less than $1.5 \mathrm{~L}$ every other day, regardless of milk production and the lactation period.

The use of the anterior urethra avoids the operation of inserting catheters into the bladder and the potential for infection. Previous studies showed better cow welfare with the anterior urine collection method than with the catheter method (Yan and Li, 1999). Fellner et al. (1988) also reported that when urine was collected via both the catheter and cup in certain experiments, the latter gave larger volumes of urine. Blood clots, which were often observed in the urine of catheterized cows, did not appear in urine collected via cups. However, the presence of certain uterine or vaginal secretions might be a problem in some applications.

Compared with the methods proposed by Hobbs et al. (1950) and Fellner et al. (1988), the apparatus avoids the gap between the meatus urethra and the urine cup, thus protecting urine from leaking down the cows' buttocks and hind legs. In addition, much labor is saved and many procedures are avoided because the materials are simple and installation is easy.

The apparatus is more economical than the methods developed by Hobbs et al. (1950) and Gorski et al. (1957). Catheters cost approximately $\$ 10.00$ each and could be used for 1 to 3 collections, and the urine cups (Fellner et al., 1988) cost about $\$ 20.00$ each and could be used for 20 to 30 collections if properly used and stored. The apparatus costs only $\$ 10.00$ (materials and labor) and could be used for 10 to 15 collections (Cao, 2007; Chen, 2007; Zhang, 2008). The uniformity of the experimental results has illustrated its effectiveness and durability.

There are many types of apparatuses for collecting urine from cows. Most of the methods are indispensable in certain situations. The greatest advantages of the apparatus in our study are the creation of an anterior urethra, which effectively protects urine collection from leakage and contamination, and straps, which keep the urine cup secured in place during different physiological activities.

The critical points in constructing the apparatus are that the large upper diameter of the anterior urethra should be a suitable size to cover the entire edge of the external genitalia, and that the straps should be wide enough to ensure that the pressure will be properly distributed without causing any injury to the body. Both factors must be determined by the anatomy of each cow. In addition, a septum cut from the glove, which is attached with an adhesive to the upper external genitalia, has been shown to avoid fecal contamination effectively and to extend the durability of adhesion to the anterior urethra.

The size of the anterior urethra and urine cup could be altered to the appropriate size for heifers. A smaller modified urine-collection apparatus has been used successfully on heifers (Dong, 2008).

\section{CONCLUSIONS}

An apparatus that can allow easy collection of urine under normal conditions for metabolism experiments with cows and heifers has been described. It is simple, inexpensive, portable, and reusable.

\section{ACKNOWLEDGMENTS}

This work was financed by the earmarked fund for Modern Agro-industry Technology Research System (Beijing, China) and the earmarked fund for Public Industry, Ministry of Agriculture (Beijing, China). The authors thank Xi Bai for the cooperation and the staff of the China Agricultural University dairy experiment farm.

\section{REFERENCES}

Balch, C. C., S. Bartlett, and V. W. Johnson. 1951. Apparatus for the separate collection of feces and urine from cows. J. Agric. Sci. 41:1715-1722.

Cao, Z. J. 2007. Effects of ration particle size and feeding management on chewing activity and metabolism of dairy cows and nutrition evaluation of distillers grains with solubles. PhD Diss., China Agricultural University, Beijing, China.

Chen, P. 2007. Study of Different Processing Oilseeds on Milk Performance and Milk Fatty Acid Regulation in Dairy Cows. China Agricultural University, Beijing, China. 
Dong, Y. X. 2008. The utilization of nitrogen and phosphorus in lactating cows in north of China. MS thesis. China Agricultural University, Beijing, China.

Fellner, V., M. F. Weiss, A. T. Belo, R. L. Belyea, F. A. Martz, and A. H. Orma. 1988. Urine cup for collection of urine from cows. J. Dairy Sci. 71:2250-2255.

Forbes, E. B., J. W. Bratzler, A. Black, and W. W. Braman. 1937. The digestibility of rations by cattle and sheep. Bull. 339. Pennsylvania Agric. Exp. Sta., University Park.

Gorski, J., T. H. Blosser, F. R. Murdock, A. S. Hodgson, B. K. Soni, and R. E. Erb. 1957. A urine and feces collecting apparatus for heifers and cows. J. Anim. Sci. 16:100-109.
Hobbs, C. S., S. L. Hansard, and E. R. Barrick. 1950. Simplified methods and equipment used in separation of urine from feces eliminated by heifers and steers. J. Anim. Sci. 9:565-570.

Yan, X. L., and A. Li. 1999. Effect of glue on the improvement method of urine collection. Chin. J. Anim. Sci. 5:17.

Zhang, H. T. 2008. The security evaluation of pure melamine and byproducts on dairy cattle. MS Thesis. China Agricultural University, Beijing, China. 